\title{
Quantum Measurements and the $\kappa$-Poincaré Group
}

\author{
Abel Camach中* \\ Department of Physics \\ Universidad Autónoma Metropolitana-Iztapalapa \\ Apartado Postal 55-534, \\ C.P. 09340, México, D.F., México. \\ A. Camacho-Galván才 \\ DEP-Facultad de Ingeniería \\ Universidad Nacional Autónoma de México.
}

(Dated: November 20, 2018)

\begin{abstract}
The possible description of the vacuum of quantum gravity through the so called $\kappa$-Poincaré group is analyzed considering some of the consequences of this symmetry in the path integral formulation of nonrelativistic quantum theory. This study is carried out with two cases, firstly, a free particle, and finally, the situation of a particle immersed in a homogeneous gravitational field. It will be shown that the $\kappa$-Poincaré group implies the loss of some of the basic properties associated to Feynman's path integral. For instance, loss of the group characteristic related to the time dependence of the evolution operator, or the breakdown of the composition law for amplitudes of events occurring successively in time. Additionally some similarities between the present idea and the so called restricted path integral formalism will be underlined. These analogies advocate the claim that if the $\kappa$-Poincaré group contains some of the physical information of the quantum gravity vacuum, then this vacuum could entail decoherence. This last result will also allow us to consider the possibility of analyzing the continuous measurement problem of quantum theory from a group-theoretical point of view, but now taking into account the $\kappa$-Poincaré symmetries.
\end{abstract}

Key words: $\kappa$-Poincaré Group, Path Integrals

\section{INTRODUCTION}

6]

The analysis of this large distance physics could begin with the $\kappa$-Poincaré group [4], since it could contain some of the physical features in connection with the quantum gravity vacuum [5]. The research in this direction has been, mainly, focused on the modifications of the Heisenberg algebra and on the measurability of distances, that this symmetry could render. In other words, the consequences of this group upon non-relativistic physics has been, as far as the authors know, not thoroughly, analyzed. Clearly the expected deviations from the usual behavior must be (as in other cases, for instance, gravitywave interferometers [2]) very small.

Nevertheless, the analysis of the effects, upon nonrelativistic theory, of the $\kappa$-Poincaré group could render important conclusions. Indeed, not only, some of the physics in the scale of low velocities could be understood, but also a second topic of current interest could obtain benefits from the present analysis, namely, the so called continuous quantum measurement problem [6]. In other words, we look for the possible description of the non-relativistic continuous quantum measurement problem employing the $\kappa$-Poincaré group. The analysis of

\footnotetext{
*Electronic address: acq@xanum.uam.mx

${ }^{\dagger}$ Electronic address: abel@servidor.unam.mx
}

the group-theoretical approach to this problem has already been considered [6], but clearly more work in this direction is needed.

We may justify this last possibility noting that the quantum-gravity-induced loss of quantum coherence is one of the main alternative viewpoints on quantum gravity [7]. Additionally, remembering that decoherence is related to quantum measurement [8], we may pose the following question: if the $\kappa$-Poincaré group describes some of the physical features involved in the quantum deformations of relativistic symmetries (induced by the quantum gravity vacuum [2] and if these quantum deformations imply that the measurability of distances is bounded by a root-mean square deviation that grows with time 9] (a fact that comprises the loss of quantum coherence [2], and hence related to quantum measurement), could this symmetry be used as part of the group-theoretical framework connected with the description of the continuous quantum measurement problem?

In the present work we analyze the consequences of the $\kappa$-Poincaré group upon the path integral of a nonrelativistic particle. In particular two cases will be considered, a free particle, and finally, a particle immersed in a homogeneous gravitational field. It will be shown that though, for practical purposes, the deviations from the usual dynamics are quite small, the effects of this symmetry might have far-reaching consequences. Indeed, the group property of the evolution operator, concerning the time parameter [10], is lost. Furthermore, the composition law for amplitudes of events occurring successively in time [10] breaks down. 
Additionally, it will be shown that some of the features related with the so called Restricted Path Integral Formalism (RPIF) 6] do appear in connection with the present approach. Particularly, it will become clear that the present model contains (similarly to the predictions of RPIF for the case of a particle whose position is being monitored) a quantum corridor, the one contains all the possible trajectories allowed by our starting symmetries. This last result advocates the possibility of analyzing the continuous quantum measurement problem using the $\kappa^{-}$ Poincaré group as part of the involved symmetries.

\section{PATH INTEGRALS AND $\kappa$-POINCARÉ GROUP}

\section{A. Minimal length and time in non-relativistic path integrals}

As mentioned in the previous section, our starting point will be the modified Heisenberg algebra encoded in the $\kappa$-Poincaré group [4, 5]. In particular we will consider one of the possibilities that this symmetry offers, namely

$$
\begin{gathered}
\Delta \hat{t} \Delta \hat{x}_{k} \geq \frac{\hbar}{2 \kappa c^{2}}\left|<\hat{x}_{k}>\right|, \\
\Delta \hat{p}_{l} \Delta \hat{x}_{k} \geq \frac{\hbar}{2} \delta_{l k}, \\
\Delta \hat{E} \Delta \hat{p}_{k} \geq \frac{\hbar}{2}, \\
\Delta \hat{t} \Delta \hat{p}_{k}=\frac{\hbar}{2 \kappa c^{2}}\left|<\hat{p}_{k}>\right| .
\end{gathered}
$$

The question concerning path integrals involves the modifications, if any, that (1) could render. Looking for the transition amplitude of finding, at time $t_{b}$, the particle at point $x_{b}$ (here for the sake of clarity we restrict the situation to one-dimensional motion), knowing that at time $t_{a}$ it was located at point $x_{a}$ we find $[9,10$.

$$
A=\int_{x_{a}}^{x_{b}} D x \exp \left\{\frac{i}{\hbar} \int_{t_{a}}^{t_{b}}\left[\frac{M}{2} \dot{x}^{2}+V(x)\right]\right\} .
$$

The last path integral is calculated considering the limit [9, 10] when $N \rightarrow \infty$ of

$$
\begin{gathered}
A \cong \frac{1}{\sqrt{2 i \pi \hbar \epsilon / M}}\left[\prod_{n=1}^{n=N} \int_{-\infty}^{\infty} \frac{d x_{n}}{\sqrt{2 i \pi \hbar \epsilon / M}}\right] \\
\times \exp \left\{\frac{i \epsilon}{\hbar} \sum_{n=1}^{N+1}\left[\frac{M}{2}\left(\frac{x_{n}-x_{n-1}}{\epsilon}\right)^{2}+V\left(x_{n}\right)\right]\right\} .
\end{gathered}
$$

Here $\epsilon=\left(t_{b}-t_{a}\right) / N$, and $N \rightarrow \infty \Rightarrow \epsilon \rightarrow 0$.

Clearly, the step that takes us from (6) to (5) entails the fact that we may, without any restriction, consider, simultaneously, the distance differences $x_{n}-x_{n-1}$, and the time difference $\epsilon$, as small as we wish.

This seems to contradict (1). A possible solution to this problem considers the introduction in the path integral of a minimal length, and also of a minimal time, i.e., the limit $N \rightarrow \infty \Rightarrow \epsilon \rightarrow 0$ is now forbidden, since it implies the simultaneous vanishing of time and length differences. Of course, a second possibility is that Feynman's formulation becomes invalid for distances $\left|x_{a}-x_{b}\right|$ smaller than a certain value, and in consequence (6) becomes meaningless.

Nevertheless, here the idea is to consider the first option and try to find the kind of modifications that, in the non-relativistic level of quantum theory, the $\kappa$-Poincaré group might render. We may justify the introduction of (1), in the calculation of the path integral, noting that in Feynman's formulation any trajectory (not only the classical one), joining the corresponding endpoints, is a possible trajectory for the motion of the involved particle 9, 10]. From this last argument it is easier to understand the need of considering the effects, upon the motion possibilities, that this restriction imposes. Otherwise, if we believe in the fact that the particle may move along any trajectory appearing in the path integral, then the particle could end up moving without taking into account the main symmetries of the situation.

Therefore, here we will assume:

(i) For a certain distance $\left|x_{b}-x_{a}\right|$, and a certain time difference $t_{b}-t_{a}$, there exists a natural number $N$, such that we may define $\epsilon \cong\left(t_{b}-t_{a}\right) /(N+1)$, and $l_{m} \cong$ $\left|x_{b}-x_{a}\right| / N=\left|x_{n}-x_{n-1}\right|, \forall n \in\{1,2,3, \ldots N+1\}$, with $\epsilon l_{m} \geq \frac{\hbar}{2 \kappa c^{2}}\left|<\hat{x}_{k}>\right|$.

(ii) In the functional integration related to (6) we are now not allowed to take the limit $N \rightarrow \infty$. In other words, the largest value of $n$ in (6) is determined by (i), i.e., $N$.

Though here we have a formalism very similar to Feynman's one, in our approach the introduction of (1) discards the limit $N \rightarrow \infty$. The consequences of these two conditions will become clear in the next two subsections. 


\section{B. Free particle}

Let us now consider the simplest case, the path integral for a one-dimensional free particle. Here (i) and (ii) mean that, for instance, if we consider the first Riemann integral, associated to $x_{1}$, we now are not allowed to integrate from $-\infty$ to $\infty$, since this would imply considering values of $x_{1}$ such that $\left|x_{1}-\left(x_{a}+x_{2}\right) / 2\right|<l_{m}$, a fact that violates (i). Hence the integration limits, associated to $x_{1}$, run from $-\infty$ to $\left(x_{a}+x_{2}\right) / 2-l_{m}$ and from $\left(x_{a}+x_{2}\right) / 2+l_{m}$ to $\infty$. In other words, those values of $x_{1}$, lying within the interval $\left[\left(x_{a}+x_{2}\right) / 2-l_{m},\left(x_{a}+x_{2}\right) / 2+l_{m}\right]$, are not considered in the functional integration. This argument is used in the $N$ integrals connected with (6). Performing the integrations we find

$$
\begin{array}{r}
A_{(f)}=\sqrt{\frac{M}{2 i \pi \hbar\left(t_{b}-t_{a}\right)}} \exp \left\{\frac{i M}{2 \hbar\left(t_{b}-t_{a}\right)}\left(x_{b}-x_{a}\right)^{2}\right\} \\
\times\left[1-\sqrt{\frac{2 l_{m} v_{m}}{i \pi \lambda c}} F\left(\frac{i l_{m} v_{m}}{\lambda c}\right)\right]\left[1-\sqrt{\frac{3 l_{m} v_{m}}{2 i \pi \lambda c}} F\left(\frac{3 i l_{m} v_{m}}{4 \lambda c}\right)\right] \\
\times \ldots \times\left[1-\sqrt{\frac{(N+1) l_{m} v_{m}}{N i \pi \lambda c}} F\left(\frac{(N+1) i l_{m} v_{m}}{2 N \lambda c}\right)\right] .(7)
\end{array}
$$

In (7) $\lambda$ denotes the Compton wavelength of the involved particle, $v_{m}=l_{m} / \epsilon$, while $F(z)$ represents the hypergeometric function $H(\alpha ; \beta ; z)$ 11], with the conditions $\alpha=1 / 2$ and $\beta=3 / 2$.

\section{Homogeneous gravitational field}

Let us now consider a more realistic case, a particle immersed in a homogeneous gravitational field described by the constant $g$. Proceeding as before we find

$$
\begin{array}{r}
A_{(g)}=A_{(f)} \exp \left\{\frac { i } { \hbar } \left[-\frac{(M g)^{2}}{24 M}\left(t_{b}-t_{a}\right)^{3} \frac{4 N+3}{4 N+4}\right.\right. \\
\left.\left.-\frac{M g}{2}\left(t_{b}-t_{a}\right)\left(x_{b}+x_{a}\right) \frac{N+2}{N+1}\right]\right\} .
\end{array}
$$

\section{Minimal length and Feynman's formulation}

Let us now analyze the case in which the minimal length $l_{m}$ vanishes. In order to do this remember 11] that if we introduce the limit $l_{m} \rightarrow 0$, then $F\left(\frac{(k+1) i l_{m} v_{m}}{2 k \lambda c}\right) \rightarrow$ 1. This last fact implies that in (7) we do recover the usual expression for a free particle [9, 10]. In the second case we also recover the path integral that stems from Feynman's idea, see equation (16.64) of [12]. In other words, the present idea contains the usual results as a limit case, when there is no minimal length.

If at this point we assume $\epsilon \sim t_{p}$, and $l_{m} \sim l_{p}$ (here $l_{p}$ and $t_{p}$ denote the Planck length and time), then $v_{m} \sim c$. For an electron we obtain $\frac{l_{m} v_{m}}{\lambda c} \sim 10^{-22}$, and hence $\left[1-\sqrt{\frac{2 l_{m} v_{m}}{i \pi \lambda c}} F\left(\frac{i l_{m} v_{m}}{\lambda c}\right)\right] \cong 1-10^{-22} / \sqrt{2}+i 10^{-22} / \sqrt{2}$. In the case when $\left|x_{a}-x_{b}\right| \sim 1 \mathrm{~cm}$, we have that $N \sim 10^{33}$, and hence the possibility of distinguishing (7) or (8) from Feynman's case lies outside the current technological capabilities.

\section{CONCLUSIONS}

In the present work we have analyzed some of the consequences of the $\kappa$-Poincaré group upon the path integral of a non-relativistic particle. In particular two cases were considered, a free particle, and finally, a particle immersed in a homogeneous gravitational field. The effects of the aforementioned symmetry were incorporated introducing, in the path integral, a minimal length, $l_{m}$, and a minimal time, $\epsilon$. The consequences of these two conditions were that now we are not allowed to integrate over all the configuration space. Additionally, the usual limit, $N \rightarrow \infty$, present in Feynman's formulation, is now forbidden.

One of the fundamental properties of Feynman's formulation comprises the composition law for amplitudes of events occurring successively in time [9, 10]. If $A\left(x_{b} ; t_{b} \mid x_{a} ; t_{a}\right)$ denotes the transition amplitude, then this composition law states [10]

$$
A\left(x_{b} ; t_{b} \mid x_{a} ; t_{a}\right)=\int A\left(x_{b} ; t_{b} \mid x_{c} ; t_{c}\right) A\left(x_{c} ; t_{c} \mid x_{a} ; t_{a}\right) d x_{c}(9)
$$

Here we have that $t_{c} \in\left[t_{a}, t_{b}\right]$. Nevertheless, within the context of the $\kappa$-Poincaré group this can not be valid, since the presence of a non-vanishing $l_{m}$ implies that now it is not allowed to integrate over the whole configuration space.

The group property, related to the time dependence, of the evolution operator, $\hat{U}\left(t_{b} ; t_{a}\right)$, is a very known fact [13], a feature that mathematically reads

$$
\hat{U}\left(t_{b} ; t_{a}\right)=\hat{U}\left(t_{b} ; t_{c}\right) \hat{U}\left(t_{c} ; t_{a}\right)
$$

where $t_{c} \in\left[t_{b}, t_{a}\right]$.

A fleeting glimpse at the usual definition of evolution operator [13] shows us that it would also be affected by $\Delta \hat{t} \Delta \hat{x}_{k} \neq 0$ 


$$
\hat{U}\left(t_{b} ; t_{a}\right)=\exp \left\{-\frac{i}{\hbar} \int_{t_{a}}^{t_{b}}\left[\frac{\hat{p}^{2}}{2 m}+V(\hat{x})\right] d t\right\} .
$$

Indeed, if we consider in (11) the case in which $t_{b}=$ $t_{a}+\epsilon / 2$, then we see that this group property breaks down, otherwise in the evolution operator $\hat{U}\left(t_{b} ; t_{a}\right)$ we would violate (i) and (ii), since we could then ask for the transition probability associated with $\left|x_{b}-x_{a}\right|=l_{m}$, and hence $\left(t_{b}-t_{a}\right)\left|x_{b}-x_{a}\right|<\frac{\hbar}{2 \kappa c^{2}}\left|<\hat{x}_{k}>\right|$. In other words, the group property associated to the time dependence of the evolution operator disappears with the case $\Delta \hat{t} \Delta \hat{x}_{k} \neq$ 0 . The extension from the one-dimensional case to a three-dimensional one is straightforward.

One of the solutions put forward in connection with the continuous quantum measurement problem comprises RPIF [6, 8]. In this approach, for the case of a particle whose position is being monitored, the main idea is the breakdown of the equiprobability of all the possible trajectories, as a consequence of the presence of the measuring device. This last fact renders the emergence of a quantum corridor, which contains all the possible trajectories that match with the measurement readout. Going back to (7) (and keeping only the lowest order in $\frac{l_{m} v_{m}}{\lambda c}$ ) it is readily seen that

$$
\begin{array}{r}
\left|A\left(x_{b} ; t_{b} \mid x_{a} ; t_{a}\right)\right|^{2}=\frac{M}{2 \pi \hbar T}\left[1-2 \sqrt{\frac{l_{m} v_{m}}{\pi \lambda c}}\right. \\
\left.-2 \sqrt{\frac{3 l_{m} v_{m}}{4 \pi \lambda c}}-\ldots-2 \sqrt{\frac{(N+1) l_{m} v_{m}}{2 N \pi \lambda c}}\right] .
\end{array}
$$

We know that in the case $l_{m}=0$ (12) entails that all the trajectories have the same probability, though, clearly, a different phase $[9,10]$. Consider now any curve $x(t)$ joining $x_{a}$ and $x_{b}$. Draw at the initial point, $x_{a}$, the straight line associated with velocity $v_{m}$. From our previous arguments (see (i) above) it is readily seen that any trajectory with initial point $x_{a}$ and lying above $v_{m}$ is not allowed. Indeed, any trajectory, joining $x_{a}$ and $x_{b}$, that contains speed values greater than $v_{m}$ defines a motion such that in a certain portion of it a distance $l_{m}$ is travelled in a time $\Delta t<\epsilon$, and in consequence it violates (i). We may rephrase this stating that the $\kappa^{-}$ Poincaré group discards some trajectories, which in the usual theory have to be included.

A fleeting glimpse at the current literature [14], in connection with the quantum measurement problem, readily shows that this aforementioned trait also appears in RPIF. This last fact allows us to consider the possibility of analyzing the continuous quantum measurement problem from a group-theoretical point of view, but now taking into account the $\kappa$-Poincaré group as part of the involved symmetries. Group methods have already been used in the study of the measurement problem [ [] , nevertheless more work in this context is needed. The results in this direction will be published elsewhere

Finally, since our approach breaks down the equiprobability of all the trajectories, we may understand the extra terms in (12) (those depending upon $l_{m} v_{m} /(\lambda c)$ ) as manifestation of decoherence, induced by the breakdown of the Poincaré symmetry. Therefore, we claim that if the vacuum of quantum gravity is described by this new symmetry [5], then it might render decoherence.

\section{Acknowledgments.}

We dedicate the present work to Alberto García on the occasion of his $60^{t h}$ birthday. This research was partially supported by CONACYT Grant 42191-F. A.C. would like to thank A.A. Cuevas-Sosa for useful discussions and literature hints.
[1] G. Amelino-Camelia, Nature 398, 216 (1999).

[2] G. Amelino-Camelia, Phys. Rev. D62, 024015 (2000).

[3] G. Amelino-Camelia, Phys. Lett. B392, 283 (1997); Mod. Phys. Lett. A13, 1319 (1998).

[4] J. Lukierski, A. Nowicki, and H. Ruegg, Ann. Phys. (NY) 243, 90 (1995).

[5] G. Amelino-Camelia, Phys. Lett. B392, 283 (1997); G. Amelino-Camelia, J. Lukierski, and A. Nowicki, Int. J. Mod. Phys. A14, 4575 (1999).

[6] M. B. Mensky, "Continuous Quantum Measurements and Path Integrals", IOP, Bristol and Philadelphia (1993). 'Quantum Measurements and Decoherence", Kluwer Academic Publishers, Dordrecht (2000).

[7] J. Ellis, J. López, N. Mavromatos, and N. E. Nanopoulos, Phys. Lett. B364, 239 (1995); P. Huet and M. E. Peskin,
Nucl. Phys. B434, 3 (1995).

[8] M. B. Mensky, "Quantum Measurements and Decoherence", Kluwer Academic Publishers, Dordrecht (2000).

[9] R. P. Feynman and A. R. Hibbs, "Quantum Mechanics and Path Integrals", Mc Graw-Hill Inc. (1965).

[10] R. P. Feynman, Rev. Mod. Phys. 20, 367 (1948).

[11] B. C. Carlson, "Special Functions of Applied Mathematics", Academic Press, New York (1977).

[12] W. Dittrich and M. Reuter, "Classical and Quantum Dynamics", Springer-Verlag, Heidelberg (1996).

[13] J. J. Sakurai, "Modern Quantum Mechanics", AddisonWesley Publishing Company, Reading, Mass. (1995).

[14] C. Presilla, R. Onofrio, and U. Tambini, Ann. Phys. (NY) 248, 95 (1996). 